\title{
A!
}

This is an electronic reprint of the original article.

This reprint may differ from the original in pagination and typographic detail.

Rayneau-Kirkhope, Daniel; Bonfanti, Silvia; Zapperi, Stefano

Density scaling in the mechanics of a disordered mechanical meta-material

Published in:

Applied Physics Letters

DOI:

$10.1063 / 1.5083027$

Published: 18/03/2019

Document Version

Publisher's PDF, also known as Version of record

Please cite the original version:

Rayneau-Kirkhope, D., Bonfanti, S., \& Zapperi, S. (2019). Density scaling in the mechanics of a disordered mechanical meta-material. Applied Physics Letters, 114(11), 1-5. [111902]. https://doi.org/10.1063/1.5083027

This material is protected by copyright and other intellectual property rights, and duplication or sale of all or part of any of the repository collections is not permitted, except that material may be duplicated by you for your research use or educational purposes in electronic or print form. You must obtain permission for any other use. Electronic or print copies may not be offered, whether for sale or otherwise to anyone who is not an authorised user. 


\section{Density scaling in the mechanics of a disordered mechanical meta-material}

Cite as: Appl. Phys. Lett. 114, 111902 (2019); https://doi.org/10.1063/1.5083027

Submitted: 27 November 2018 . Accepted: 04 March 2019 . Published Online: 18 March 2019

Daniel Rayneau-Kirkhope (iD), Silvia Bonfanti (iD), and Stefano Zapperi (D)
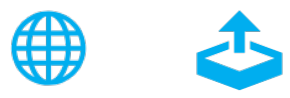

Export Citation

\section{ARTICLES YOU MAY BE INTERESTED IN}

Left-handed band in an electromagnetic metamaterial induced by sub-wavelength multiple scattering

Applied Physics Letters 114, 111101 (2019); https://doi.org/10.1063/1.5065556

Negative thermal expansion of nanoporous anodic aluminum oxide membranes

Applied Physics Letters 114, 111901 (2019); https://doi.org/10.1063/1.5074083

Robust modeling of acoustic phonon transmission in nanomechanical structures

Applied Physics Letters 114, 113101 (2019); https://doi.org/10.1063/1.5068685

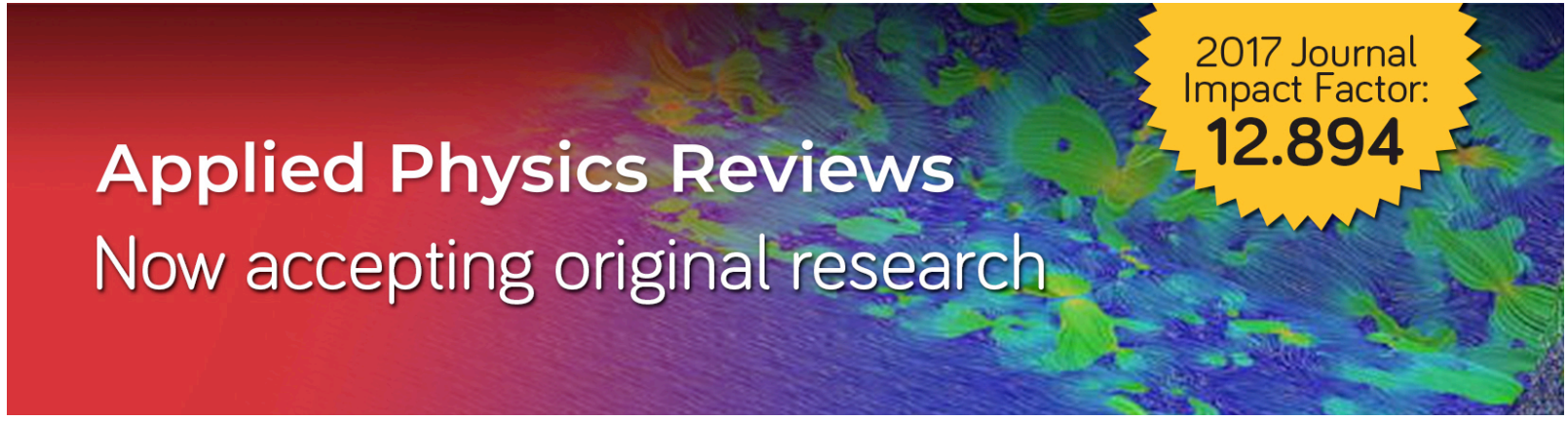




\title{
Density scaling in the mechanics of a disordered mechanical meta-material
}

\author{
Cite as: Appl. Phys. Lett. 114, 111902 (2019); doi: 10.1063/1.5083027 \\ Submitted: 27 November 2018 - Accepted: 4 March 2019 . \\ Published Online: 18 March 2019
}

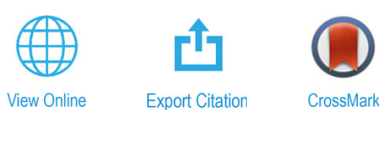

\author{
Daniel Rayneau-Kirkhope, ${ }^{7}$ (D) Silvia Bonfanti, ${ }^{2}$ (D) and Stefano Zapperi ${ }^{2,3, a)}$ (D)

\begin{abstract}
AFFILIATIONS
'Department of Applied Physics, Aalto University, P.O. Box 11100, FI-00076 Aalto, Finland

${ }^{2}$ Center for Complexity and Biosystems, Department of Physics, University of Milano, via Celoria 16, 20133 Milano, Italy

${ }^{3}$ CNR-Consiglio Nazionale delle Ricerche, Istituto di Chimica della Materia Condensata e di Tecnologie per l'Energia,

Via R. Cozzi 53, 20125 Milano, Italy
\end{abstract}

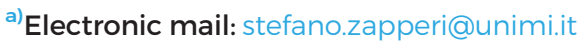

\begin{abstract}
Nature provides examples of self-assemble lightweight disordered network structures with remarkable mechanical properties which are desirable for many application purposes but challenging to reproduce artificially. Previous experimental and computational studies investigated the mechanical responses of random network structures focusing on topological and geometrical aspects in terms of variable connectivity or probability to place beam elements. However, for practical purposes, an ambitious challenge is to design new materials with the possibility to tailor their mechanical features such as stiffness. Here, we design a two dimensional disordered mechanical meta-material exhibiting unconventional stiffness-density scaling in the regime where both bending and stretching are relevant for deformation. In this regime, the mechanical meta-material covers a wide interval of the Young modulus-density plane, simultaneously exhibiting high critical stress and critical strain. Our results, supported by finite element simulations, provide the guiding principles to design on demand disordered metamaterials, bridging the gap between artificial and naturally occurring materials.
\end{abstract}

Published under license by AIP Publishing. https://doi.org/10.1063/1.5083027

Lattice materials of many kinds, such as foams, ${ }^{1}$ cellular solids, microlattices, ${ }^{3}$ and trusses, ${ }^{4}$ which are made of connected elements, have been intensively studied mainly due to their lightweight structures and remarkable mechanical properties. ${ }^{5}$ The high strength to weight ratio of bone ${ }^{6}$ or balsa, ${ }^{7}$ the elastic properties of spider silk, ${ }^{8}$ and the fracture resistance of nacre ${ }^{9}$ are just few of the naturally occurring structures that derive their mechanical properties from their underlying geometry. These types of materials attract growing interest in many fields ranging from commercial products such as those related to food industry to architectural applications such as energy absorption and management ${ }^{1}$ and in modern technologies where their geometrical features are exploited to achieve myriads of performances.

In recent years, composite structures have been started to be rationally architected with the aim of achieving targeted properties, ${ }^{10}$ and emerging significant breakthroughs ${ }^{11}$ are favoured alongside the advances in digital manufacturing technologies, i.e., 3D printing and automated assembly. Artificially designed materials have been recently termed meta-materials; ${ }^{12-14}$ specifically, mechanical meta-materials indicate a class of structures whose mechanical properties are a consequence of their underlying geometry rather than their constituent material. ${ }^{15}$ Through the prudent choice of a meta-material underlying architecture, it is possible to create geometries whose structural performance far exceeds that of the material from which it is made. ${ }^{16}$ These structures can be designed to exhibit a wide range of beneficial properties, including a high strength to weight ratio, ${ }^{17-20}$ auxetic behaviour, ${ }^{10}$ energy trapping, ${ }^{21}$ and fracture resistance, ${ }^{22}$ among many others. ${ }^{15}$

Typically, artificial meta-materials are designed with a single motif repeated periodically throughout the material, ${ }^{14}$ in contrast to the disordered structures encountered in self-assembled natural objects. ${ }^{23}$ Random elastic networks are widely studied, ${ }^{24}$ but there are still large gaps between theoretical investigations and practical purposes such as real solid materials. Mechanical meta-materials with the increasing degree of disorder are just emerging. ${ }^{25-27}$ Whether disorder is merely the price to pay for self-assembly or whether it provides some advantage is still an open issue.

The structural behaviour of lattice based meta-materials, made up of beams with non-hinged nodes, is generally divided into two regimes: stretching-dominated and bending-dominated. ${ }^{28}$ Which of these two regimes a lattice falls into is dependent on its connectivity: when a lattice's connectivity is less than that is required for rigidity in 
its rigid link freely hinged analogous system, the lattice will exhibit bending dominated behaviour, and for lattices with connectivity above this threshold, stretching dominated behaviour will be observed. ${ }^{28}$ The two classes of lattices exhibit dissimilar mechanical properties. For example, the relative stiffness of a two-dimensional lattice $(\tilde{Y} / Y)$ is related to its relative density $(\tilde{\rho} / \rho)$ via the following expression:

$$
\tilde{Y} / Y \sim(\tilde{\rho} / \rho)^{n},
$$

where, in two-dimensions, $n$ is one or three for stretching-dominated and bending dominated architectures, respectively ${ }^{29}$ (in $d=3, n$ takes the values of 1 and 2 for stretching and bending dominated architectures, respectively ${ }^{28}$ ). Due to this scaling behavior, stretchingdominated lattices of low relative density are stiffer than their bending-dominated counterparts. In the above expressions, $Y$ and $\rho$ are the Young's modulus and density of the construction material, respectively, while $\tilde{X}$ denotes the property $X$ of the meta-material. Density dependent scaling laws similar to the one reported in Eq. (1) can also be established for other mechanical properties, such as buckling or strength, or even electrical properties.

Inspired by naturally designed disordered structures, in this letter, we consider the linear response and elastic limit of disordered lattices, showing that they exhibit an intermediate regime characterized by an unconventional density scaling and many advantageous mechanical properties. At low densities, the lattice far exceeds the bending dominated lattice in terms of stiffness. At higher densities, the mixed regime simultaneously shows a high buckling load and critical strain. With the increasing manufacturing freedom to design structures on the micro- and nano-scale ${ }^{30}$ and the possibility of self-assembly of complex architectures, ${ }^{31}$ the theoretical work presented here is of increasing technological relevance.

To investigate the density scaling of disordered meta-materials, we consider a disordered triangular lattice: this lattice is generated by taking a perfect triangular lattice and removing links at random such that each linkage has a probability of $1-p$ being removed. Each linkage element is constructed of a slender beam of thickness $t$ and length $L$, made of a linear elastic material with Young's modulus $Y$ and Poisson's ratio $\nu$. We define the aspect ratio of the beam to be $a=L / t$. From the resultant architecture, we take the elements of the lattice which make up the largest connected component (the largest sublattice in which any pair of nodes is connected by a path along a sequence of beam elements ${ }^{32}$ ) and investigate the mechanical properties of the resultant meta-material. The system size is such that the entire lattice measures $N_{x} L$ horizontally and $\sqrt{3} L N_{y} / 2$ vertically (see supplementary material). The designed geometries are subsequently deformed under a strain in the negative $y$ direction (in all cases, the imposed strain is less than the critical strain). The thickness of the beams was varied in order to vary the relative density.

All simulations presented here have been performed using COMSOL Multiphysics ${ }^{33}$ and COMSOL with MATLAB ${ }^{33}$ in 2dimensions using a plane strain approximation through the use of the structural mechanics module. All studies assume a linear elastic material with Young's modulus of $50 \mathrm{MPa}$ and Poisson's ratio of 0.3 ; the length of the beams used was $0.01 \mathrm{~m}$. Mesh refinement studies were undertaken to ensure convergence of results. The results pertaining to stiffness have been obtained using Euler-Bernoulli beam elements using the in-built "stationary studies" (a quasi-static solver). Here, a small displacement was applied to the upper boundary, while the lower boundary was fixed in place inducing a reduction in the total height of the structure, the remaining boundaries were left free; the reaction force required to induce this displacement was then calculated, and from this, an effective Young's modulus was calculated. For studies on buckling strength and critical strain, the lower boundary was again fixed, while the upper boundary had an applied force or displacement imposed (for buckling stress and strain, respectively), the remaining boundaries were left free. For these studies, the solid mechanics module used alongside the in-built linear buckling solvers (linear eigenvalue solver). For studies using the solid mechanics module, mesh density required was highly dependent on the aspect ratio of the beams; approximately 200-1500 elements were used per unit cell.

Figure 1(a), 1(b), and 1(c) shows an example of three geometries for $p=0.4,0.642,0.95$, respectively, deformed along the negative $y$ direction. Beams are colored according to the proportion of bending energy with respect to the total. ${ }^{34}$ Increasing $p$, the designed random meta-materials cover different ranges from bending to stretching behavior. Figure 1(b) shows that for a dilute triangular lattice, close to the transition from the stretching dominated in the bending dominated regime $\left(p=p_{\mathrm{T}}\right.$, in this work $p_{\mathrm{T}}$ is taken to be 0.642 in line with previous estimates ${ }^{35}$ ), an intermediate scaling regime is observed where contributions to stiffness from both stretching and bending cannot be ignored. This results in a new exponent $n \simeq 2$ that lies between the value $n=1$ expected for stretching dominated elasticity and $n=3$ for bending dominated elasticity.

To obtain a theoretical estimate of the density scaling of a disordered lattice, we can use effective-medium theory (EMT), ${ }^{36}$ which considers in a self-consistent manner the interaction of one element
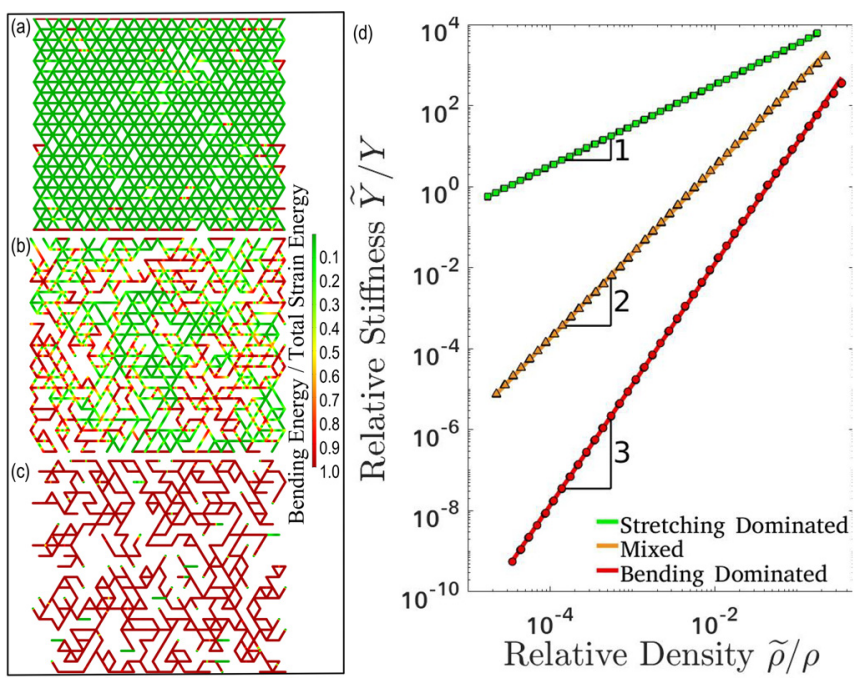

FIG. 1. Different scaling regimes of stiffness vs relative density. Representative examples of the various regimes for a small system of size $20 \times 20$ with a small strain imposed in the $y$ direction. The colors show the proportion of the local strain energy that is stored as bending energy with red indicating bending energy that dominates and yellow showing mixed and green indicating stretching behaviour. (d) Stretching $(n=1)$, mixed $(n=2)$, and bending $(n=3)$ dominated lattices are characterised by power law behaviours, and fitting of the data shown gives scaling of $n=2.97 \pm 0.02,2.1 \pm 0.05$, and $1.00 \pm 0.01$ for bending, mixed, and stretching, respectively. Data refer to $128 \times 128$ node networks reported in the supplementary material. 
with the average of the others. Under the EMT approximation, the contribution to stiffness will follow ${ }^{37} \tilde{Y} \sim \mu^{(1-x)} \kappa^{x}$, where $\mu$ and $\kappa$ are the stretching and bending stiffness of the lattice elements, respectively. EMT predicts that $x=0.5$ in $d=2$ and $x=0.4$ in $d=3$, where $d$ is the dimensionality of the system. ${ }^{37}$ For a lattice made up of beam elements of thickness $t$ and length $L$, we note that $\mu \sim(t / L)^{q}$ and $\kappa \sim(t / L)^{r}$, where for $d=2, q=1$ and $r=3$, while for $d=3, q=2$ and $r=4$. Since $\tilde{\rho} / \rho \propto(t / L)$ in $d=2$ and $\tilde{\rho} / \rho \propto(t / L)^{2}$ in $d=3$ within the intermediate regime, we expect to observe the scaling, $\tilde{Y} / Y \sim(\tilde{\rho} / \rho)^{n}$, with $n=2$ in $d=2$ and $n=1.4$ in $d=3$. This scaling is plotted against the results of simulation for probabilities close to the critical probability $p_{\mathrm{T}}$ in Fig. $1(\mathrm{~d})$. This intermediate scaling close to the isostatic point is reminiscent observed in stochastic fibre networks where stretching and bending energies can be varied arbitrarily. In contrast, in this work, the geometric parameters of the beam elements set the ratio of bending to stretching energies in the beam elements, ${ }^{38}$ and thus, here, we observe a dependence of the stiffness of the metamaterial on its relative density.

In contrast to the behaviour of fibre networks which exhibit a "bending rigidity percolation" probability which can be calculated through Maxwell counting and mean field arguments, ${ }^{39}$ here, we observe that the connectivity of the upper and lower boundaries is sufficient to yield a non-zero stiffness in a stochastic meta-material constructed from beam elements. Thus, we see non-zero effective Young's modulus for probabilities greater than the geometric percolation probability $p_{c}$ which for the triangular lattice is given by ${ }^{40} p_{c}=2 \sin (\pi / 18)$.

For $p-p_{c} \ll 1$, we find that the Young's modulus obeys standard finite size scaling expected for percolation

$$
\tilde{Y} \sim N_{x}^{-s / \nu} f\left(\left(p-p_{c}\right) N_{x}^{1 / \nu}\right),
$$

where $\nu=4 / 3$ is the correlation length exponent, while $s$ is the elasticity exponent that can be expressed in terms of the conductivity exponent $t \simeq 0.98 \nu^{41}$ as $s=t+2 \nu \simeq 4{ }^{42}$ Equation (2) is verified by the data collapse reported in Fig. 2 for three different lattice sizes with $N_{x}=64,128,256$.
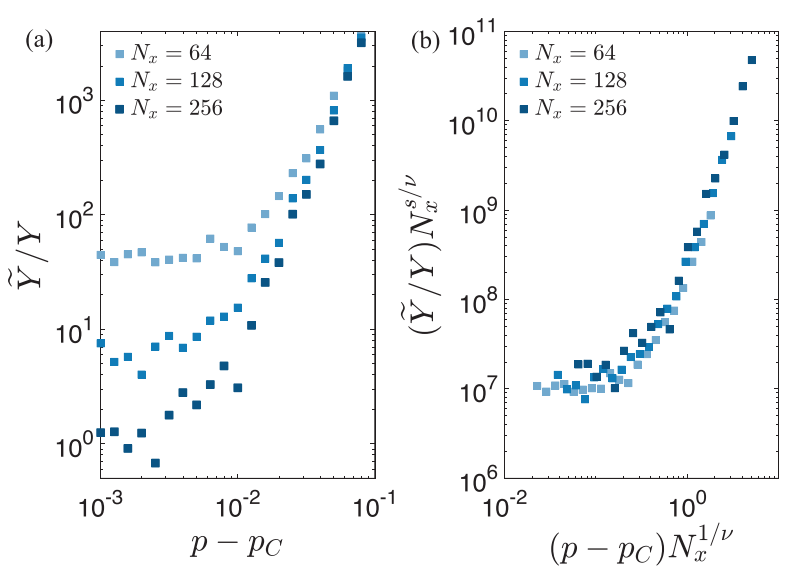

FIG. 2. (a) The Young modulus $\tilde{Y}$ as a function of $p-p_{c}$ for different values of $N_{x}$. The data shown are for beams with an aspect ratio of 16 . Each data point shown is an average over a number of simulations $N_{s}$ such that the product of $N_{s}$ and the number of elements in the perfect system is greater than $10^{6}$. (b) Rescaling of the data in (a) according to Eq. (2).
We summarize these findings in Fig. 3 where we show the onset of non-zero stiffness at $p_{c}$ and the region of $(p, a)$-space for which the bending, stretching, and mixed regimes are observed. With decreasing slenderness of beams (high relative densities), a wider range of $p$ values lead to lattices where both stretching and bending energies must be considered.

In the stochastic metamaterial made up of slender beam elements, elastic instability will occur when the loading reaches a critical value. Here, we investigate the dependence of this critical stress/strain on relative density and the connectivity of the lattice through analytical and computational techniques. To investigate this phenomenon computationally, we use COMSOL Multiphysics ${ }^{33}$ utilising the solid mechanics module with a built-in linear buckling (eigenvalue) solver. This requires a finer mesh for convergence, and thus, only small system sizes can be simulated. It is expected that when $d=2$ or $d=3$, the critical value will scale as $\sigma_{c} \sim F_{c} / L^{d-1} \sim Y I / L^{d+1}$, where $\sigma_{c}$ is the critical stress, $F_{c}$ is the Euler buckling force of a slender beam, $I$ and $L$

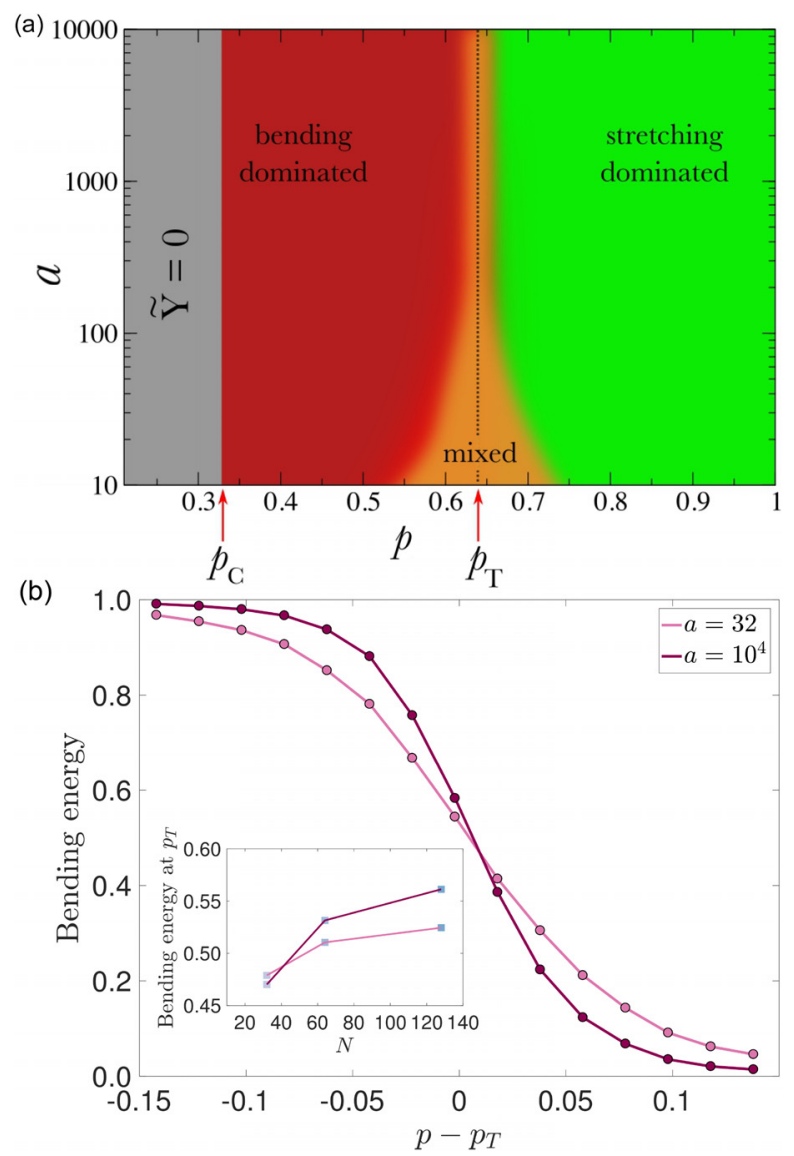

FIG. 3. (a) The parameter space $(p, a)$ and the observed regime of stretching and bending dominated alongside a mixed regime. (b) The transition from bending (low $p$ ) to stretching (high $p$ ) dominated regimes for system size $N_{x}=128$ for systems made up of beams with aspect ratios of 32 and $10^{4}$; a sharper transition is seen for the higher aspect ratio of beams. The inset shows the variation of the bending energy as a function of three system sizes, $N_{x}=32,64,128$. The main panel shows averages over a number of simulations $N_{s}$ chosen in such a way that the product of the total number of elements in the perfect system $(p=1)$ and $N_{s}$ is greater than $2 \times 10^{5}$. 
are the second moment of area and length of the component beams, respectively, and $Y$ is the Young's modulus of the construction material. Thus, through variation of the aspect ratio of the component beams with a fixed $p$, we expect a scaling relationship between critical stress and density given by

$$
\sigma_{c} \sim(\tilde{\rho} / \rho)^{m},
$$

where $m=3$ when $d=2$ and $m=2$ when $d=3 .^{28}$ This is confirmed in Fig. 4 where the scaling of $m=3$ is found to be irrespective of the regime (stretching, bending, or mixed) to which the lattice belongs. The critical strain (the imposed strain that will induce buckling, $\Delta_{c}$ ) can also be calculated from simulation, and for the meta-material

(a)

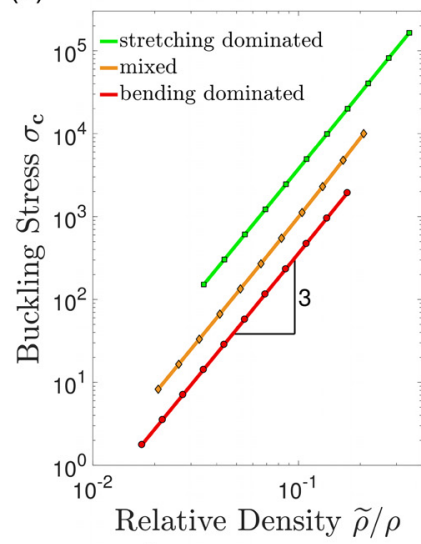

(b)

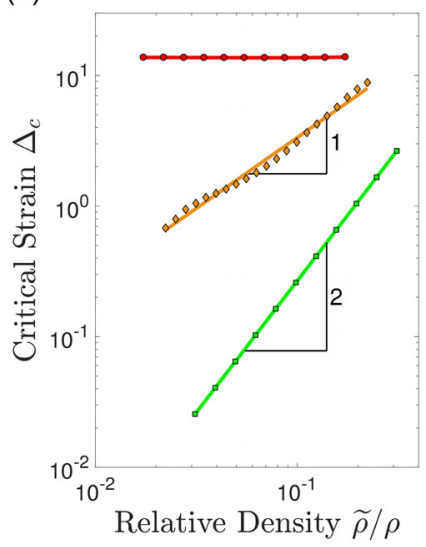

(c)

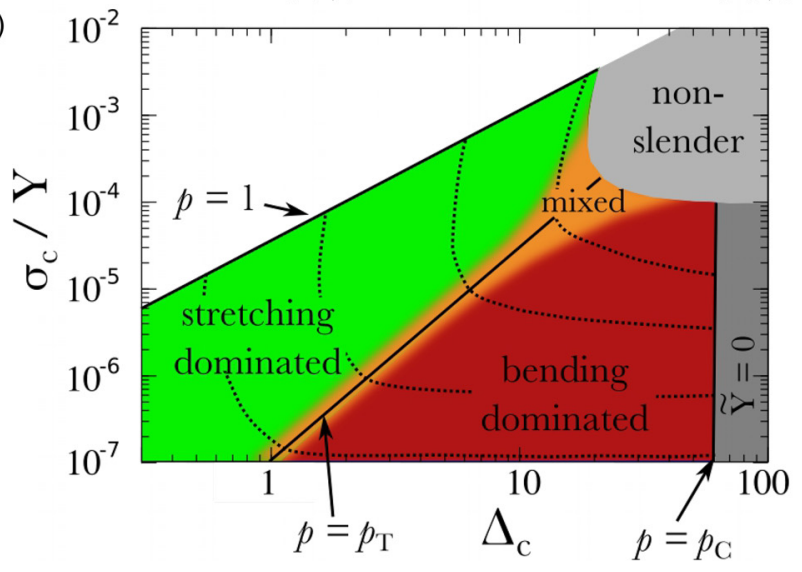

FIG. 4. (a) The critical stress for lattices in the bending, mixed, and stretching regimes. All regimes show the same scaling of $\sigma_{c}$ vs relative density as predicted using Eq. (3). (b) The critical strain for the stochastic metamaterial for the different regimes; here, the scaling is dependent on the regime and is predicted using Eq. (4). (c) Stretching dominated regimes lead to high critical stress with low critical strain, and bending dominated lattices exhibit high critical strain but low critical stress; Lattices with significant energy contributions from both bending and stretching simultaneously exhibit both high stress and strain at failure. The data shown are for a system size of $N_{x}=N_{y}=20$. The various phases shown in (c) are established through simulation: we establish the critical strain and critical stress of a given architecture for a given $p$ varying the aspect ratio of the constituent beams. The dotted contour lines show lines of constant beam aspect ratio, while lines of constant $p$ are solid lines. Each regime is established from the scaling of critical strain against relative density. considered, it will obey $\sigma_{c}=\tilde{Y} \Delta_{c}$. For a given material, therefore, combining Eqs. (1) and (3), we can calculate the dependence of critical strain on the relative density of the lattice. It is predicted here that $\Delta_{c}$ will follow the scaling:

$$
\Delta_{c} \sim(\tilde{\rho} / \rho)^{(m-n)},
$$

where when $d=2, m=3$ irrespective of the regime and $n$, as in Eq. (1), takes the values of 1,2 , or 3 when the lattice is stretching dominated, mixed, or bending dominated, respectively (in $d=3, m=2$ irrespective of the regime, and $n$ is expected to take the value of $1,1.4$, or 2 for stretching dominated, mixed, or bending dominated, respectively); this prediction for $d=2$ is confirmed in Fig. 4. We thus find that both high relative stiffness and high relative strength are achieved through the use of the mixed deformation mode, that is, $p \approx p_{\mathrm{T}}$, which is shown in Fig. 4. We note that for lattices with $p=1$ (all lattice elements present), the lattices exhibit a global failure mode, ${ }^{43}$ this is in marked contrast to the cases for $p<1$ where local buckling is observed in the regions with least strength. In both cases however $(p=1$ and $p<1$ ), it is observed that a plateau in the stress-strain curve is observed in the vicinity of the buckling load, with the transition to plateau in stress becoming more localised for higher $p$ (see supplementary material).

Lattice based meta-materials, made up of beam elements, have been traditionally classified as either bending or stretching dominated depending on their connectivity. ${ }^{28}$ It is notable that recent experimental work has established that some 3 dimensional lightweight nanolattices exhibit density scaling that is inconsistent with these classifications. ${ }^{44}$ Here, we have established, through theory and simulation, the existence of a third class of lattice architecture where both bending and stretching energies must be considered, leading to metamaterials with high critical stress and critical strain. This third class of lattice is expected to be present regardless of the geometry of the lattice investigated: When the connectivity makes the transition from stretching to bending dominated lattice through the random removal of bonds, it is expected that this intermediate regime is present. Whether randomness of bond removal is a requirement to create this regime remains an open question. Stochastic design is not the only strategy to obtain unconventional density scaling, as shown in the past using hierarchical lattices. $^{18-20,34,45}$

Better understanding of stochastic lattice based structures has importance for a range of applications from the performance of lightweight structures to early diagnosis of diseases. For example, osteoporosis, a disease with major socio-economic consequence, is linked with changes both in bone mineral density (BMD) and in the connectivity of the trabecular bone latticework. ${ }^{46}$ Understanding the implications of density and connectivity in stochastic lattices is thus a key step for the early diagnosis of this disease. ${ }^{46}$

It is notable that this stochastic methodology generates structures that are closer in design to their naturally occurring counterparts. These naturally occurring structures exhibit high flaw tolerance and insensitivity to perturbations. ${ }^{23}$ Although this flaw tolerance is linked with optimisation of the material geometry and hierarchy, ${ }^{23}$ it is plausible that disorder could play a role. Such a link between randomness and robustness has been previously found in machine resilience, ${ }^{47}$ algorithm design, ${ }^{48}$ and interdependent lattice networks. ${ }^{49}$ Extending this link to structural mechanics has huge potential for the design of functional robust structures. 
See supplementary material for additional details on the geometry of the lattice, details on buckling, and additional configurations (supplementing Fig. 1).

D.R.-K. would like to acknowledge funding support from the Academy of Finland postdoctoral grant program. S.Z. acknowledges support from the Academy of Finland FiDiPro program, Project No. 13282993. S.Z. and S.B. are supported by the project DISORDER funded by the Ministero degli Affari Esteri e della Cooperazione Internazionale.

\section{REFERENCES}

${ }^{1}$ M. Ashby, Philos. Trans., A 364, 15 (2006).

${ }^{2}$ L. J. Gibson and M. F. Ashby, Cellular Solids: Structure and Properties (Cambridge University Press, 1999).

${ }^{3}$ N. Fleck, V. Deshpande, and M. Ashby, Proc. R. Soc. London, A 466, 2495-2516 (2010).

${ }^{4}$ V. S. Deshpande, N. A. Fleck, and M. F. Ashby, J. Mech. Phys. Solids 49, 1747 (2001).

${ }^{5}$ L. J. Gibson, J. Biomech. 38, 377 (2005).

${ }^{6}$ R. Hodgskinson and J. Currey, J. Mater. Sci.: Mater. Med. 3, 377 (1992).

${ }^{7}$ X. Zheng, H. Lee, T. H. Weisgraber, M. Shusteff, J. DeOtte, E. B. Duoss, J. D.

Kuntz, M. M. Biener, Q. Ge, J. A. Jackson et al., Science 344, 1373 (2014).

${ }^{8}$ H. Zhou and Y. Zhang, Phys. Rev. Lett. 94, 028104 (2005).

${ }^{9}$ H. Kakisawa and T. Sumitomo, Sci. Technol. Adv. Mater. 12, 064710 (2011).

${ }^{10}$ R. Lakes, Science 235, 1038 (1987).

${ }^{11}$ T. Frenzel, M. Kadic, and M. Wegener, Science 358, 1072 (2017).

${ }^{12}$ T. Bückmann, N. Stenger, M. Kadic, J. Kaschke, A. Frölich, T. Kennerknecht, C. Eberl, M. Thiel, and M. Wegener, Adv. Mater. 24, 2710 (2012).

${ }^{13}$ J. Paulose, A. S. Meeussen, and V. Vitelli, Proc. Natl. Acad. Sci. 112, 7639 (2015).

${ }^{14} \mathrm{~K}$. Bertoldi, V. Vitelli, J. Christensen, and M. van Hecke, Nat. Rev. Mater. 2, 17066 (2017).

${ }^{15}$ T. J. Cui, D. Smith, and R. E. Liu, Metamaterials: Theory, Design, and Applications (Springer US, 2010).

${ }^{16}$ D. Rayneau-Kirkhope, C. Zhang, L. Theran, and M. A. Dias, Proc. R. Soc., A 474, 20170753 (2018).

${ }^{17}$ X. Yu, J. Zhou, H. Liang, Z. Jiang, and L. Wu, Prog. Mater. Sci. 94, 114 (2017).

${ }^{18}$ D. Rayneau-Kirkhope, Y. Mao, and R. Farr, Europhys. Lett. 119, 14001 (2017).

${ }^{19}$ R. M. Hensleigh, H. Cui, J. S. Oakdale, J. C. Ye, P. G. Campbell, E. B. Duoss, C. M. Spadaccini, X. Zheng, and M. A. Worsley, Mater. Horiz. 5, 1035 (2018).

${ }^{20}$ X. Zheng, W. Smith, J. Jackson, B. Moran, H. Cui, D. Chen, J. Ye, N. Fang, N. Rodriguez, T. Weisgraber, and C. M. Spadaccini, Nat. Mater. 15, 1100- 11EP (2016).
${ }^{21}$ S. Shan, S. H. Kang, J. R. Raney, P. Wang, L. Fang, F. Candido, J. A. Lewis, and K. Bertoldi, Adv. Mater. 27, 4296 (2015).

${ }^{22}$ P. Das, J.-M. Malho, K. Rahimi, F. H. Schacher, B. Wang, D. E. Demco, and A. Walther, Nat. Commun. 6, 5967 (2015).

${ }^{23}$ P. Fratzl, J. R. Soc. Interface 4, 637 (2007).

${ }^{24}$ S. Feng and P. N. Sen, Phys. Rev. Lett. 52, 216 (1984).

${ }^{25}$ M. M. Driscoll, B. G.-G. Chen, T. H. Beuman, S. Ulrich, S. R. Nagel, and V. Vitelli, Proc. Natl. Acad. Sci. 113, 10813 (2016).

${ }^{26}$ M. Hanifpour, C. F. Petersen, M. J. Alava, and S. Zapperi, Eur. Phys. J. B 91, 271 (2018).

${ }^{27}$ D. R. Reid, N. Pashine, J. M. Wozniak, H. M. Jaeger, A. J. Liu, S. R. Nagel, and J. J. de Pablo, Proc. Natl. Acad. Sci. 115, E1384 (2018).

${ }^{28}$ I. J. Gibson and M. F. Ashby, Proc. R. Soc. London. A. Mathematical and Physical Sciences 382, 43 (1982).

${ }^{29}$ D. Rayneau-Kirkhope, Sci. Rep. 8, 12437 (2018).

30 J. Bauer, L. R. Meza, T. A. Schaedler, R. Schwaiger, X. Zheng, and L. Valdevit, Adv. Mater. 29, 1701850 (2017).

${ }^{31}$ C. N. Kaplan, W. L. Noorduin, L. Li, R. Sadza, L. Folkertsma, J. Aizenberg, and L. Mahadevan, Science 355, 1395 (2017).

${ }^{32}$ F. Harary, Graph Theory (Addison-Wesley, Reading, MA, 1969).

${ }^{33}$ COMSOL Multiphysics, COMSOL, AB, 39, 2012.

${ }^{34}$ R. Oftadeh, B. Haghpanah, D. Vella, A. Boudaoud, and A. Vaziri, Phys. Rev, Lett. 113, 104301 (2014).

${ }^{35}$ S. Roux and A. Hansen, Europhys. Lett. 6, 301 (1988).

${ }^{36}$ S. Kirkpatrick, Rev. Mod. Phys. 45, 574 (1973).

${ }^{37}$ C. P. Broedersz, X. Mao, T. C. Lubensky, and F. C. MacKintosh, Nat. Phys. 7, 983 (2011).

${ }^{38}$ S. P. Timoshenko and J. M. Gere, Dover Civil and Mechanical Engineering (Dover Publications, New York, NY, 2009).

${ }^{39}$ C. P. Broedersz and F. C. MacKintosh, Rev. Mod. Phys. 86, 995 (2014).

${ }^{40}$ M. Sykes and J. Essam, Phys. Rev. Lett. 10, 3 (1963).

${ }^{41}$ P. Grassberger, Physica A 262, 251 (1999).

${ }^{42}$ M. Sahimi, J. Phys. C: Solid State Phys. 19, L79 (1986).

${ }^{43}$ S. H. Kang, S. Shan, A. Košmrlj, W. L. Noorduin, S. Shian, J. C. Weaver, D. R. Clarke, and K. Bertoldi, Phys. Rev. Lett. 112, 098701 (2014).

${ }^{44}$ L. R. Meza, G. P. Phlipot, C. M. Portela, A. Maggi, L. C. Montemayor, A. Comella, D. M. Kochmann, and J. R. Greer, Acta Mater. 140, 424 (2017).

${ }^{45}$ A. Vigliotti and D. Pasini, Mech. Mater. 62, 32 (2013).

${ }^{46}$ C. S. Rajapakse, J. S. Thomsen, J. S. E. Ortiz, S. J. Wimalawansa, E. N. Ebbesen, L. Mosekilde, and G. H. Gunaratne, J. Biomech. 37, 1241 (2004).

${ }^{47}$ M. Randles, D. Lamb, E. Odat, and A. Taleb-Bendiab, J. Comput. Syst. Sci. 77, 293 (2011)

${ }^{48}$ P. Hertling, C. M. Hoffmann, W. Luther, and N. Revol, Reliable Implementation of Real Number Algorithms: Theory and Practice. International Seminar Dagstuhl Castle, Germany, January 8-13, 2006, Revised Papers (Springer, 2008), Vol. 5045.

${ }^{49}$ J. Yuan, L. Li, H. Peng, J. Kurths, J. Xiao, and Y. Yang, Chaos 26, 013105 (2016). 